\title{
Pregnancy Induced Hypertension among Pregnant Women in Dhaka City, Bangladesh
}

\author{
Md. Mahedi Hasan', Md. Nahian Rahman², Md. Mahbubur Rahman², Sabrina Rahman ${ }^{3}$ \\ ${ }^{I}$ BSc (Institute of Nutrition and Food Science, Dhaka University, Bangladesh), MPH (Northern University), ${ }^{2} M S c$, \\ Institute of Nutrition and Food Science, University of Dhaka, Bangladesh, ${ }^{3}$ BSc (Institute of Nutrition and Food \\ Science, Dhaka University), MPH (North South University)
}

\begin{abstract}
This cross-sectional study has been conducted in the Bangabandhu Sheikh Mujib Medical University Gynaecology and Obstetrics Division on 72 patients who have been admitted from December 2018 to March 2019, in order to find out the percentage of pregnancy-induced high blood pressure among pregnant mothers. Of the 72 patients, $25(34.7 \%)$ have been diagnosed with pre-eclamptictoxaemia $27(37.1 \%)$ induced pregnancy and 20(27.8\%) with eclampsia. Most people were 21-25 years old and their average age was 25.3 years old. Of the 72 patients, 38 were primipara (52.8 percent). 20 (27.8\%) of newborns were delivered before 37 weeks, while $52(72,2 \%)$ were delivered before 37 weeks. A cesarean section was available in 37 of all patients (51 percent). The cesarean portion in PIH \& PET was higher. Of the 72 newborn patients 16(22.2\%) were low in birth weight, and patients with ecclampsy were more likely to experience low birth weight. 35 of 72 patients had poor fetal results, including 1.4 percent perinatal and 33.3 percent asphyxiated infants. Only 5 (6.5 percent) had complications in maternal outcomes such as strokes, renal eclampsia. All participants in this study are women who are pregnant and receive prenatal care. WHO normally recommends patients with an antecedent of antenatal treatment less than 4 times eclampsia. In patients with eclampsia it is more common to see low birth weight, pre-term delivery and the complication for prenatal mortality.
\end{abstract}

Keywords: Primipara, ecclampsia, asphyxiated infants, hypertension, blood pressure.

\section{Introduction}

One of the most common complications of birth and one of the most common causes for fetal and maternal morbidity and death worldwide, hypertensive pregnancy disorder[1]. Almost 10 per cent of all births are complicated by elevated blood pressure and the frequency is greater if women are nulliparous or have several fetuses[1]. The pattern to complicate multigrain loss is if proper management is not achieved in good time [2].

\section{Corresponding Author:}

Md. Mahedi Hasan

MPH, BSc in Nutrition, Sharifpur, National

University-1704, 34 No Ward, Gazipur City, Gazipur, Bangladesh

e-mail: mdmahedi.hasan.official@gmail.com

Mobile: +88 01838506406
One of the main causes of maternal death and morbidity is hypertensive pregnancy disorders in Bangladesh. Details on the occurrence of this condition would be documented on a prescribed schedule, including blood pressure, albuminuria, edema, immunization and antenatal treatment. Even though the effects of these conditions have been minimized in developed countries by adequate prenatal care on the mothers and fetuses, it endangers the result of pregnancies in most developing countries such as Bangladesh. Lack of education, a lack of sufficient antenatal care and nutrition has caused births more likely to suffer the symptoms of hypertensive disorders. Bangladesh, which also has an exceptionally high rate of maternal mortality due to preventable reasons, is a country with a high population density. In Bangladesh, death from mothers is a significant issue of public health[3].

Hypertensive maternity disease involves gestational high blood pressure, gestational uric hypertension protein, and chronic high blood pressure. The risk of 
high blood sugar disorders, including preterm birth, restrained intrauterine development, perinatal mortality, acidic renal and hepatic dysfunction, anteriorpartum hemorrhage, postpartums hemorrhage (Postpartum hemorrhage) including epilepsy, stroke, premature delivery, and maternal death, is complicated by $5-10$ percent across all pregnancies [3].

Maternal or fetal outcomes of hypertensive pregnancy disorders rely on various factors particularly if the early pregnancy is high in hypertension. Hypertension caused by pregnancy, which involves both hypertension and preeclampsia in infancy, a common and morbid complication of pregnancy. Emerging data indicates that insulin resistance associated with critical hypertension may play a role in hypertension triggered by pregnancy. Conditions associated with increasing insulin resistance may be predisposed to hypertensive pregnancy, including gestational diabetes, ovary syndrome, and obesity [5].

The group of symptoms concerned with elevated blood pressure, proteinuria and convulsion during breastfeeding are the hypertensive pregnancy disorders. Preeclampsia and eclampsia have the most severe effects on mother and infant. Eclampsia is typically the product of the preeclampsia of a central nervous system and is often unconscious and can lead to death if untreated. Both preeclampsia and eclampsia are not well evaluated in their long term sequelae and mortality is the cause of hypertensive diseases in the pregnancy[6].

Hypertension $(\mathrm{Sbp}>140 \mathrm{mmHg}$ or DBP $11>90$ $\mathrm{mmHg})$ and proteinuria $(0.3 \mathrm{~g}$ protein in 24 -hour or $1+$ on a dipstick) after 20 weeks of gestation for the previously normoteness women lead to new onset of hypertension (SBPs $>140 \mathrm{mmHg}$ or DBPs $11>90$ $\mathrm{mmHG})$. It is a life-threatening, multi-organ illness that appears to be the main cause of maternal mortality. Its clinical manifestations are due to widespread vasospasm, coagulation system triggering and changes in the volume-related and blood pressure-control humoral and autoregulatorysystems[7].

High perinatal death and morbidity rates are blamed for pre-ecampsia, largely due to the early end of pregnancy. The symptoms of chronic placental hypo-perfusion[7] are fetus growth limitation, oligohydramniotics, and no comforting fetal status.

Preeclampsia is significant in the developed as well as in the developing world. It also remains a leading cause of death and morbidity of pregnancy and perinatal conditions. With a prior history of preeclampsia, preexisting diabetes, multiple births, family history of preeclampsia [3], the likelihood of preeclampsia is enhanced.

Pregnancy with high maternal and perinatal mortality is highly dangerous and relatively normal. Antenatal appointments should be screened in order to guard from the hazards of preeclampsia pregnant. Bangladesh is one of the developed countries that lack functional antenatal care services and is also the leading cause of maternal and perinatal mortality in developing countries. Although ecclampsy has become increasingly avoidable and uncommon in developing countries, in Bangladesh it remains a major problem in obstetrics [6].

In Bangladesh, Eclampsia accounts for $16 \%$ of maternal death. It remains the leading cause in many other areas of the world of maternal and perinatal mortality, including Bangladesh. In developing countries this is a common problem, where analphabets, lack of knowledge, health education, poverty and superstition preclude women from seeking healthcare during pregnancy. There is a lack of sufficient maternal treatment in developed countries such as Bangladesh. The coverage of prenatal care (minimum 1 visit) in Bangladesh is $48.7 \%$ [6].

While exact etiology is still unknown, the incidence of complications with proper antenatal care can be avoided. There are many disorders in our country because of poor prenatal care, early marriage and more pregnancy. This can reduce the complication by early detection and treatment. With this backdrop, this study was designed to determine the hypertensive disorders of pregnant women in Bangladesh.

Justification of the study: Hypertension is one of the common complications of pregnancy, contributing significantly to maternal and perinatal mortality and morbidity.

Hypertension is a symptom of chronic condition pre-existing or arising during birth for the first time. The recognition and successful control of this therapeutic organization play a decisive role in the result of both mother and baby pregnancy. Many births exist unrestricted in developed countries such as Bangladesh, where there are many prospects unnoticed before significant complications arise. 
Early marriage in this country is more commonly seen in the underprivileged sector and hypertensive condition \& associated complication is also popular. It may cause severe maternal and fetal problems or losses if it is untreated and neglected. This study shows the maternal and fetal results of a patient with high blood pressure. This study findings can be used to increase awareness among hypertensive moms, public health professionals, clinicians and health officials who can use an intervention program to minimize mortality and morbidity in mothers and fetuses due to the effects of hypertension.

The research is intended to provide useful knowledge on hypertension during pregnancy and the pregnancy of the high-volume mother to medical staff, public health professionals, average citizens and hypertensive mothers. In order to produce a better result, the individual involved would have a preventive action at a reasonable moment, effectively mitigating the unfortunate fate, reducing maternal and fetal mortality and morbidity.

\section{Materials and Method}

\section{Study Objectives}

1. General Objective:To estimate the proportion of pregnancy induced hypertension among pregnant women.

\section{Specific objective}

1. To find out the complication of hypertension among respondents.

2. To find out the factors responsible for hypertension.

3. To find out the type of hypertension disorder in pregnancy.

3. Study design: It was a descriptive type of crosssectional study.

4. Study population:Pregnant woman with hypertension (either pre-existing or pregnancyinduced) delivered baby at the obstetric department of Bangabandhu Sheikh Mujib Medical University irrespective of age, gravida.

5. Sample Population: The sample population was those who were available at the time of data collection.

6. Study Site: Bangabandhu Sheikh Mujib Medical
University which is a tertiary level hospital in Dhaka.

7. Study Area: Bangabandhu Sheikh Mujib Medical University which is a tertiary hospital in Dhaka. The hospital established in 1965 which situated at Shahbag, Dhaka. This study is done in the Obstetrics and Gynecology Department, This department situated in building Block-C (8th Floor). The emergency unit of the obstetric department is situated in building Block-D (ground floor).

8. Study Period: Total Study lasted for a period of four months commencing from December 2018 to March 2019.

9. Sample size: Statistical calculation of sample size was followed by using a sound statistical formula, indicated below:

$$
n=\frac{Z^{2} p q}{d^{2}}
$$

Where, $\mathrm{n}=$ Desired sample size, $\mathrm{Z}=$ Standard normal deviate, usually set at 1.96

which corresponds to $95 \%$ confidence level.

$\mathrm{p}=30.2 \%=0.312[10]$

$\mathrm{q}=1-\mathrm{p}=(1-0.312)=0.688$

$\mathrm{d}=$ Degree of absolute precision, usually at $0.5 \%$

Therefore, the desired number of participants is

$$
n=\frac{(1.965)^{2} * 0.312 * 0.688}{(0.025)^{2}}=329.84
$$

The sample size was taken to be 72 after discussion with the supervisor of the study.

10. Inclusion \& exclusion criteria: Inclusion criteria-Having the ability to understand interview questions.-Having the ability to give information.

Exclusion Criteria: Those who are seriously ill or unwilling to talk to the interviewer would be excluded from the study.

11. Sampling technique: A purposive sampling technique was used

12. Data collection tools: A questionnaire was prepared and printed. The final questionnaire was used for data collection. Hospital-records were also reviewed.

13. Data collection technique: Face to face interview, observation and record review. A brief introduction was given verbally to each respondent by the 
interviewer at the beginning of the interview to explain the purpose and importance of the study. The questionnaire was filled up by the interviewer during the interview. Socio-demographic, previous reproductive information was collected by the interviewer.

14. Data management \& analysis: All the data were checked, cleaned and edited after collection. Then those cleaned data were entered into the SPSS program of computer were done by the SPSS-20 program on the computer. Analyses were done according to the objectives and variables of the study. Simple techniques of data analysis, as for example-frequency, percentage, average etcwere done by the SPSS program in the computer and results were presented in the form of tables and graphs.

\section{Limitations of the study:}

1. As it is a hospital record-based study of BSMMU, the findings of the study about antenatal checkup may not reflect the actual picture of the entire Bangladesh

2. The patients who did not bring their antenatal care visit card or who had no obstetric checkup records were not included in the study population.

3. Many of the respondents did not give their previous history of pregnancy-induced hypertension.

4. As I have used some administrative recordbased data which may include women with incorrect information.

5. Shortage of time for data collection was the main limitation.

6. Small sample size was one of the weaknesses of this study.

7. The sampling technique was used in this study was purposive sampling which had chances of bias.

\section{Result and Discussion}

Hypertension can be graded as chronic if recognised before 20 weeks of gestation, or if it happens only in the second half of pregnancy. This distinction is clinically beneficial since almost all hypertension arising in the first half of pregnancy results from underlying chronic hypertension. The progression of hypertension in the second half of pregnancy is more complicated, arising from either a pregnancy-specific phase or a complex interplay of pregnancy with renal failure or chronic hypertension leading to exacerbation of hypertension[9].

The research sought to see the result of women with hypertensive disorder during pregnancy and to figure out the respondents' obstetric background and sociodemographic characteristics. This is a cross-sectional descriptive style of research and was performed at Bangabandhu Sheik Mujib Medical University among pregnant women who were admitted to the eclampsia and labour ward for delivery for antenatal checkups in the antenatal treatment outpatient unit. Pregnant women aged 20 weeks or older who had medical tests during this pregnancy were included. Bangladesh's maternal mortality rate is 3.2 per thousand live births. Eclampsia is the third-largest source of maternal mortality. Many preeclamptic and gestational pregnant women eventually experience eclampsia and complications and even death.

WHO reports that 15 percent of women have a degree of hypertension during pregnancy.Fortunately, most of these cases are benign, not requiring medication or complication. However in some cases women have a hypertensive pregnancy condition like pre-eclampsia, which can lead to severe complications or death. Hypertensive pregnancy results in $12 \%$ global maternal mortality and up to $40 \%$ maternal death in some countries[11].

72 pregnant women with hypertensive conditions were studied. Much research population is from lowincome communities. Of 72 patients, $32 \%$ had monthly income ranging from 8,000 to 10,$000 ; 16.7 \%$ had monthly family income $>10,000 ; 15.3 \%$ had s 6,000 ; and only $6.9 \%$ had $<4,000$ taka. [Table 1 ].

Much of the sample population refers to the age group 25 years and are more likely to experience hypertension than the other age group. To compare this study with another previous study, a Relationship with Parity study like this shows that the majority (52 percent) was primiparous; simulating with other studies at home and abroad. Regarding the level of education, 26 percent are in high school, 44.41 percent are in elementary school, 25 percent are illiterate. Buchbinder et al 19 observed $25.4 \pm 5.3$ in patients with moderate preeclampsia and $25.9 \pm 6$ years with extreme preeclampsia. Relationship with parity indicates that 71.5 percent of the majority 
are primipara, with 26.4 percent in high school being 44 percent in elementary school, 25 percent analphabet. [Table 1].

\section{Table 1: Distribution of respondents by Demographic information}

\begin{tabular}{|c|c|c|}
\hline Variables & Frequency & Percentages \\
\hline \multicolumn{3}{|l|}{ Age } \\
\hline$\leq 20$ years & 9 & 12.5 \\
\hline $21-25$ years & 26 & 36.1 \\
\hline 26-30 years & 20 & 27.8 \\
\hline$>30$ years & 17 & 23.6 \\
\hline \multicolumn{3}{|l|}{ Religion } \\
\hline Muslim & 62 & 86.1 \\
\hline Hindu & 10 & 13.9 \\
\hline \multicolumn{3}{|l|}{ Education } \\
\hline Illiterate & 18 & 25 \\
\hline Primary & 32 & 44.4 \\
\hline Secondary & 19 & 26.4 \\
\hline Degree and Above & 3 & 4.2 \\
\hline \multicolumn{3}{|l|}{ Occupation } \\
\hline House Wife & 27 & 34.7 \\
\hline Agriculture & 23 & 31.7 \\
\hline Service & 22 & 30.6 \\
\hline \multicolumn{3}{|c|}{ Husbands Occupation } \\
\hline Agriculture & 24 & 33.3 \\
\hline Service & 22 & 30.6 \\
\hline Business & 16 & 22.2 \\
\hline Rickshaw puller & 9 & 12.5 \\
\hline Others & 1 & 1.4 \\
\hline
\end{tabular}

\begin{tabular}{|l|c|c|}
\hline Variables & Frequency & Percentages \\
\hline Family Income & 21 & 29.2 \\
\hline$\leq 4000$ & 5 & 6.9 \\
\hline $4001-6000$ & 11 & 15.3 \\
\hline $6001-8000$ & 23 & 31.9 \\
\hline $8001-10000$ & 12 & 16.7 \\
\hline$>10000$ & & \\
\hline Family Member & 32 & 44.4 \\
\hline$<4$ & 34 & 47.2 \\
\hline $4-6$ & 6 & 8.3 \\
\hline $7-8$ & \multicolumn{3}{|l|}{} \\
\hline
\end{tabular}

The number of family members ranges from 4-6 in most patients. According to national statistics, the family member is 2.3 and the goal is 3.5. People of specified occupations were found, most of them housewives. [Table: 1]

Among the sample group, P.E.T. suffered mostly. (37.1\%), followed by PIH (34.3\%), and Eclampsia (27.8\%). [Figure: 1]

Most participants in this sample received prenatal treatment, this care has an increasingly important role to play in reducing maternal and perinatal morbidity and mortality because pregnancy hypertension cannot be prevented, but usually maternal death can. Considering antenatal treatment, 80.6 percent of the overall population obtained antenatal care independent of maternity forms of hypertensive disorders. $73 \%$ of patients received antenatal treatment, so it is clear that there could be a link between less antenatal care and eclampsia. Factors identifying between low-risk and high-risk women experiencing hypertensive symptoms and recognizing the condition as early as possible to schedule rational antenatal treatment and maternal-fetal monitoring [8].

\section{Type of Hypertension disorder during Prengnancy}

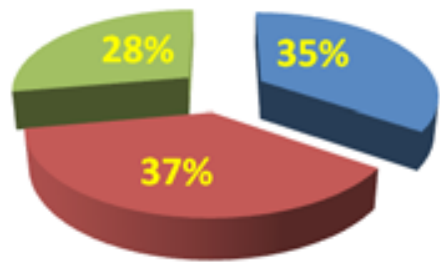

= Pregnancy induced hypertension

Figure 1: Distribution of respondents by type of hypertension disorder during pregnancy 
Table 2: Distribution of respondents by complication during pregnancy period

\begin{tabular}{|l|c|c|}
\hline Variables & Frequency & Percentages \\
\hline Edema Present & 43 & 59.7 \\
\hline Yes & 29 & 40.3 \\
\hline No & 5 & 6.9 \\
\hline Proteinurea Present & 29 & 40.3 \\
\hline Yes & 38 & 52.8 \\
\hline No & 20 & 27.8 \\
\hline Don't Know & 52 & 72.2 \\
\hline Experience convulsion & 1 & 1.4 \\
\hline Yes & 71 & 98.6 \\
\hline No & 52 & 72.2 \\
\hline Unconscious & 20 & 27.8 \\
\hline Yes & \multicolumn{2}{|l}{} \\
\hline No & \multicolumn{2}{|l|}{} \\
\hline Family History of hypertension & \\
\hline Yes & \multicolumn{2}{|l|}{} \\
\hline No & \multicolumn{2}{|l|}{} \\
\hline
\end{tabular}

Table 3: Problems during pregnancy

\begin{tabular}{|l|c|c|}
\hline Variables & Frequency & Percentages \\
\hline Abortion & 27 & 37.5 \\
\hline Yes & 45 & 62.5 \\
\hline No & 6 & 8.3 \\
\hline Stillbirth & 66 & 91.7 \\
\hline Yes & 6 & 8.3 \\
\hline No & 66 & 91.7 \\
\hline Neonatal Death & \multicolumn{1}{|l}{} \\
\hline Yes & 6 & \\
\hline No &
\end{tabular}

A typical complication of hypertensive disease is prematureness, either due to spontaneous labour or obstetric activity of interrupting pregnancy due to impaired maternal-fetal. A research in our area showed a higher relative risk of prematurity in pregnant women with chronic hypertension compared to a pregnancyinduced population. Prematurity raises perinatal morbidity and mortality rates of potential imminent or late sequels, requiring public policy to protect these neonates [5].

Analyzing fetal result, it was clear that 31(51.9\%) were alive and well, 24 births. (33.1\%) were birth- asphyxiated, 13\% were stillborn, 1(1.4\%) died after birth.

In the present study, fetal outcome in eclampsia patients was comparatively poor than the other 2 classes. Considering low birth weight was 16 . On the other birth weight, 28\%. Hand-cumulative evidence from another retrospective analysis revealed a birth-weight incidence of $28 \%[2]$.

The present research found that $32.3 \%$ of babies were born before 37 completed weeks; the remainder was delivered after 37 weeks. There is no substantial correlation in pregnant women between prior experience of hypertension and hypertensive disorder. There is no clear link between pregnant women's family history of hypertension and hypertensive disorder.

A recent study found that both gestational and chronic hypertension increased the relative risk of premature SAG, limited intrauterine growth, and low birth weight were the results of premature delivery among women with severe pre-eclampsia[5].

In this report, $50 \%$ of babies were born via cesarean section; most of them had PET and PIH. In eclampsia patients, cesarean section was comparatively fewer than the other 2 categories.

Of 72 patients, 46.5 percent) had different complications and all were eclampsia patients. This group of patients had no maternal mortality. Of 72 patients, 20 experienced convulsion. Of 20 patients experiencing seizures, $75 \%$ of seizures occur at home and $25 \%$ of seizures occur in hospital.

Of the 72 patients, only 20 patients took hypertension medication, and 52 patients took no hypertension medication.

Since the study was limited to a few patients admitted to Bangabandhu Sheik Mujib Medical's gynecology and obstetrics department. University over a brief period of time, the research might have little epidemiological significance, yet it definitely provides an aggregate picture of hypertensive condition patients in our nation.

\section{Conclusion}

The births all come from all social backgrounds and were aged 18 - 39 years. In this study, the age of the pregnant woman was linked with hypertension, which was higher than the respondent's age and higher. 
All pregnant women should be appropriately trained, appropriate technologies should be used to recognise risk factors, and appropriate antenatal treatment and care should be given for them during childbirth. Sensitizing and emphasizing the risk factors within the population on health issues is an important step in controlling hypertensive disease during pregnancy. This can be achieved in multiple mainstream media

The family history of elevated blood pressure and the hypertension of the respondents during this pregnancy are not closely related.

\section{Reference}

1. National High Blood Pressure Education Program; working group report on high blood pressure in pregnancy.(Am J ObsterGynecol 1990; 163: 16891712.)

2. Hussain F, Bhuiyan AB. Pregnancy Outcome in Hypertensive Disorders of Pregnancy in DMCha study of 100 cases, (Bangladesh Heart Journal 2014; 9(2): 53-56.)

3. Brown MA. The detection, investigation and management of hypertension in pregnancy: full consensus statement. (Aust NZJ ObsterGynaecol 2000; 40: 139-155)
4. American heart association web-https://www. hyper.ahajournal.org/egi/contant/full/36/2/149

5. Janquira SM. Topic- PIH and neonatal outcome 11/6/2007, 2018;21(1): 53-8

6. BBS.Statistical pocketbook of Bangladesh. Bangladesh Bureau of Statistics, Statistics Division, Ministry of Planning, Dhaka, 2012

7. Journal of obstetrics and Gynaecology Canda. 2008; 30(3): 18-19.

8. Allen VM. The effect of hypertensive disorders in pregnancy on small for gestational age and stillbirth: a population based study. BMC Pregnancy and chilbirth 2004, 4:17http://www.biomedcentral. com/1471-2393/4/17

9. Chanprapaph P. update in pre-eclampsia. J Med Thai. 2014; 87(3): s104-12

10. Chanprapaph P. update in pre eclampsia. J Med Thai. 2014; 87(3): s104-12

11. Detection and Management of Hypertensive Disorders of Pregnancy. (Maternaland Neonatal Health. Available from: URL:http://www.mnh. jhpiego.org). 